Document downloaded from:

http://hdl.handle.net/10251/104015

This paper must be cited as:

Bru García, R.; Gasso Matoses, MT.; Gimenez Manglano, MI.; Santana-De Asis, MDJ. (2017). Diagonal entries of the combined matrix of a totally negative matrix. Linear and Multilinear Algebra. 65(10):1971-1984. doi:10.1080/03081087.2016.1261079

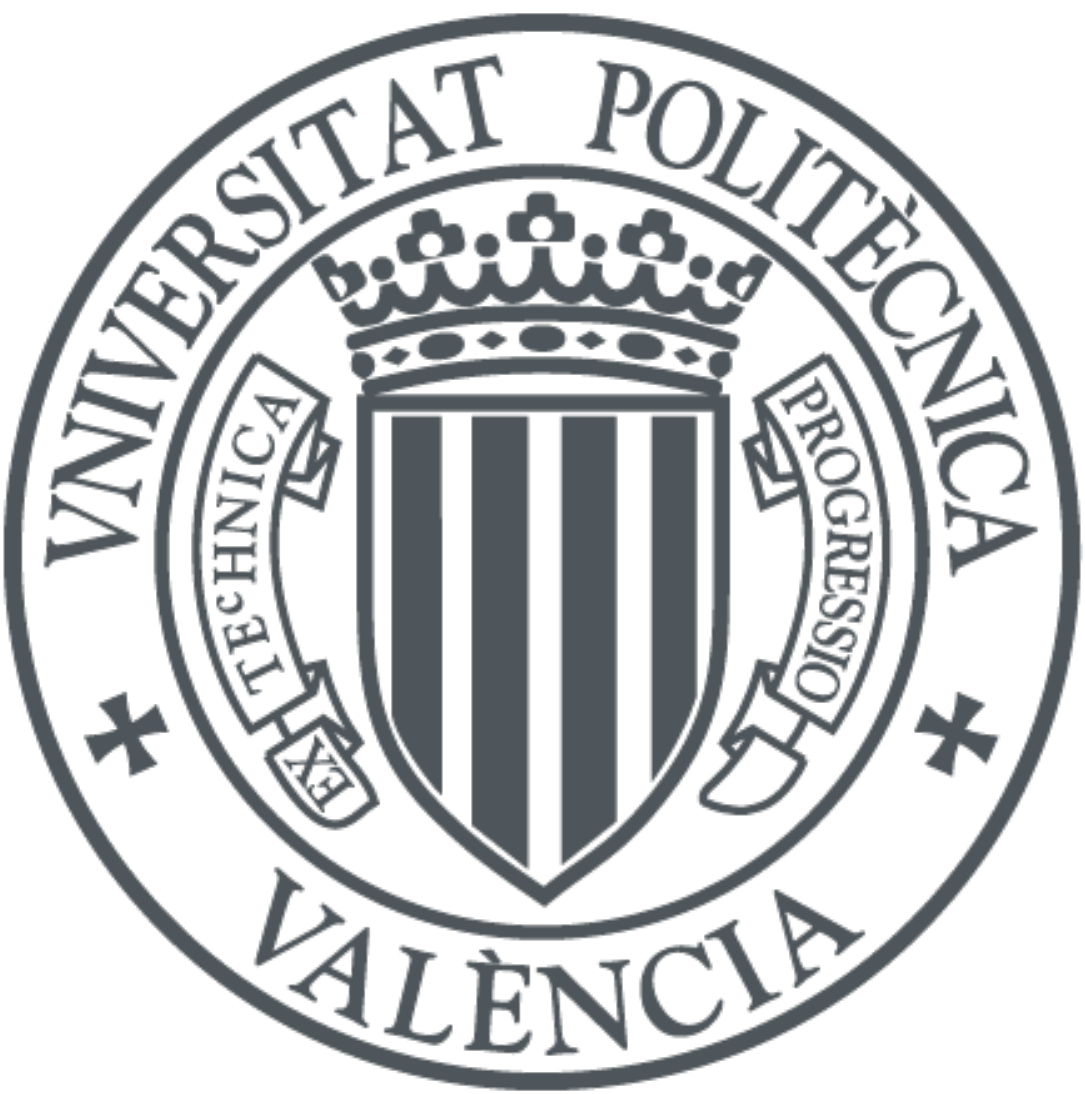

The final publication is available at

https://doi.org/10.1080/03081087.2016.1261079

Copyright Taylor \& Francis

Additional Information 


\title{
Diagonal entries of the combined matrix of a totally negative matrix *
}

\author{
Rafael Bru, Maria T. Gassó^, Isabel Giménez ${ }^{\star}$ \\ and Máximo Santana ${ }^{\star \star}$

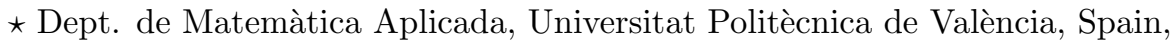 \\ rbru/mgasso/igimenez@mat.upv.es \\ $\star \star$ Inst. de Matemática, Universidad Autónoma de Santo Domingo, Dominican Rep., \\ msantana22@uasd.edu.do
}

\begin{abstract}
The combined matrix of a nonsingular matrix $A$ is the Hadamard (entrywise) product $A \circ\left(A^{-1}\right)^{T}$. This paper deals with the characterization of the diagonal entries of a combined matrix $C(A)$ of a given nonsingular real matrix $A$. A partial answer describing the diagonal entries of $C(A)$ in the positive definite case was given by Fiedler in 1964. Recently in 2011, Fiedler and Markham characterized the sequence of diagonal entries of the combined matrix $C(A)$ for any totally positive matrix $A$ when the size is 3 . For this case, we characterize totally negative matrices and we find necessary and sufficient conditions for the sequence of diagonal entries of $C(A)$, in both cases, symmetric and nonsymmetric.
\end{abstract}

Keywords: Totally negative matrices, Hadamard product, relative gain array, combined matrix, stochastic matrices

AMS Subject Classification: 15A24; 15B48

*Research supported by Spanish DGI grant number MTM-2014-58159-P and Dominican Republic FONDOCYT grant number 2015-1D2-166. 


\section{Preliminaries}

This paper deals with the characterization of the diagonal entries of the combined matrix $C(A)$ of a given real matrix $A$. A partial answer describing the diagonal entries of $C(A)$ in the positive definite matrix case was given by Fiedler [9]. Recently, Fiedler and Markham [11] characterized the sequence of the diagonal entries of the combined matrix $C(A)$ for any totally positive matrix $A$ of size 3 . In this work, we study this problem for totally negative matrices.

All matrices in this paper are real. Let us recall the basic definitions and properties. Some recent works on combined matrices are given in [3] for positive and negative matrices, in [4] for sign regular matrices and in [5] for $\mathrm{H}$-matrices. Test to recognize total positive matrices has been studied in $[15]$.

We recall that the combined matrix $C(A)$ of a nonsingular matrix $A$ is defined as $C(A)=A \circ\left(A^{-1}\right)^{T}=A \circ A^{-T}$, where $\circ$ is the Hadamard (entry wise) product, $(A \circ B)_{i j}=a_{i j} b_{i j}$. The combined matrix $C(A)$ has the property that $\sum_{j} c_{i j}=\sum_{i} c_{i j}=1$ for rows and columns. As a consequence, if $C(A) \geq$ 0 , then $C(A)$ is a doubly stochastic matrix. In [13] the interesting relationship among diagonal entries and eigenvalues of a diagonalizable matrix is given. Moreover, if $A$ represents the gain of a control process, $C(A)$ represents the relative gain of the process [2]. This last representation can be applied to economic or chemical problems.

A property of combined matrices we use later is the following: If we multiply the matrix $A$ by a nonsingular diagonal matrix from the left or from the right, its combined matrix $C(A)$ does not change.

We recall that a matrix $A$ is totally positive (negative) if all its minors of any order are positive (negative). That is, if for every subsets $\alpha, \beta \subseteq N$ : $\operatorname{det}(A[\alpha, \beta])>0(\operatorname{det}(A[\alpha, \beta])<0)$. They are denoted by TP (t.n.).

We use the special submatrices defined in [10]. A submatrix of $A$ of size $r \times s$ is called relevant if has $r$ consecutive rows and the first $s$ columns or if it has the first $r$ rows and $s$ consecutive columns, where $r, s \in N$.

Theorem 1.1 ([6]) Let $A$ be a $n \times n$ matrix. $A$ is totally negative if and only if the minor of every relevant submatrix of $A$ is negative.

In the next section we extend the results for totally positive matrices, given by Fiedler and Markham in [11], to the totally negative case. We find 
necessary and sufficient conditions for the sequence of diagonal entries of $C(A)$ if $A$ is a $3 \times 3$ totally negative matrix.

\section{Results}

Note that in case $n=2$,

$$
A=\left[a_{i j}\right]=\left[\begin{array}{ll}
a_{11} & a_{12} \\
a_{21} & a_{22}
\end{array}\right] \rightarrow A^{-1}=\left[\alpha_{i j}\right]=\frac{1}{\operatorname{det} A}\left[\begin{array}{cc}
a_{22} & -a_{21} \\
-a_{12} & a_{11}
\end{array}\right]
$$

and

$$
a_{11} \alpha_{11}=a_{22} \alpha_{22}, \quad a_{12} \alpha_{12}=a_{21} \alpha_{21} .
$$

Therefore, we establish the following lemmas only for $n \geq 3$.

Lemma 2.1 Let $A=\left[-a_{i j}\right]$ be an $n \times n$ totally negative matrix. Let $n \geq 3$ and $B$ is a $k \times k, k \leq n$, submatrix of $A$. Then there exists a $k \times k$ matrix $Q$ with the same entries of $B$ but the entry $-a_{k k}$ is changed by $-\hat{a}_{k k}$ with $\hat{a}_{k k}>a_{k k}$ and such that $\operatorname{det} Q=0$.

Proof: Without loss of generality, let $k=n$ and then $B=A$. Denote the $(i, j)$ cofactor of $B$ by $C_{i j}$. Then,

$$
-a_{1 k} C_{1 k}-a_{2 k} C_{2 k}-\cdots-\left(a_{k k}+\frac{\operatorname{det} B}{C_{k k}}\right) C_{k k}=0 .
$$

Constructing the matrix $Q$ of the lemma choosing

$$
-\hat{a}_{k k}=-a_{k k}-\frac{\operatorname{det} B}{C_{k k}},
$$

we have $\hat{a}_{k k}>a_{k k}$, since $B$ es totally negative, and $C_{k k}<0$. Note that the left hand side of (1) is $\operatorname{det} Q$, therefore, $\operatorname{det} Q=0$. 
Lemma 2.2 Let $A=\left[-a_{i k}\right]$ be an $(n-1) \times n$ totally negative matrix. If $n \geq 3$ and $a_{11}=a_{12}$, then

$$
\begin{array}{r}
\operatorname{det}\left[\begin{array}{cccc}
-a_{11} & -a_{13} & \cdots & -a_{1 n} \\
-a_{21} & -a_{23} & \cdots & -a_{2 n} \\
\vdots & \vdots & & \vdots \\
-a_{n-1,1} & -a_{n-1,3} & \cdots & -a_{n-1, n}
\end{array}\right] \\
\\
<\operatorname{det}\left[\begin{array}{cccc}
-a_{12} & -a_{13} & \cdots & -a_{1 n} \\
-a_{22} & -a_{23} & \cdots & -a_{2 n} \\
\vdots & \vdots & & \vdots \\
-a_{n-1,2} & -a_{n-1,3} & \cdots & -a_{n-1, n}
\end{array}\right] .
\end{array}
$$

Proof: We use induction on $n$. If $n=3$, the matrix $A$ has the form

$$
A=\left[\begin{array}{lll}
-a_{11} & -a_{12} & -a_{13} \\
-a_{21} & -a_{22} & -a_{23}
\end{array}\right]
$$

with $a_{11}=a_{12}$. Since $A$ is totally negative, we have

$$
\operatorname{det}\left[\begin{array}{ll}
-a_{11} & -a_{12} \\
-a_{21} & -a_{22}
\end{array}\right]<0
$$

and then $a_{22}<a_{21}$. Hence

$$
\operatorname{det}\left[\begin{array}{ll}
-a_{11} & -a_{13} \\
-a_{21} & -a_{23}
\end{array}\right]<\operatorname{det}\left[\begin{array}{ll}
-a_{12} & -a_{13} \\
-a_{22} & -a_{23}
\end{array}\right] .
$$

Suppose now that $n>3$ and that for $(n-2) \times(n-1)$ size the result holds.

We are going to apply Lemma 2.1 to obtain the submatrix $Q$ such that

$$
\operatorname{det} Q=\operatorname{det}\left[\begin{array}{cccc}
-a_{12} & -a_{13} & \cdots & -a_{1 n} \\
-a_{22} & -a_{23} & \cdots & -a_{2 n} \\
\vdots & \vdots & & \vdots \\
-a_{n-1,2} & -a_{n-1,3} & \cdots & -\hat{a}_{n-1, n}
\end{array}\right]=0
$$

and $\hat{a}_{n-1, n}>a_{n-1, n}$. For that, given an $\epsilon>0$, the matrix

$$
F_{\epsilon}=\left[\begin{array}{cccc}
-a_{11} & -a_{12} & \cdots & -a_{1 n} \\
-a_{21} & -a_{22} & \cdots & -a_{2 n} \\
\vdots & \vdots & & \vdots \\
-a_{n-1,1} & -a_{n-1,2} & \cdots & -\hat{a}_{n-1, n}+\epsilon
\end{array}\right]
$$


has the same relevant submatrices that $A$ except for the submatrix

$$
R_{\epsilon}=\left[\begin{array}{cccc}
-a_{12} & -a_{13} & \cdots & -a_{1 n} \\
-a_{22} & -a_{23} & \cdots & -a_{2 n} \\
\vdots & \vdots & & \vdots \\
-a_{n-1,2} & -a_{n-1,3} & \cdots & -\hat{a}_{n-1, n}+\epsilon
\end{array}\right]
$$

Observe that $\operatorname{det} R_{\epsilon}$ is equal to

$\operatorname{det}\left[\begin{array}{cccc}-a_{12} & -a_{13} & \cdots & -a_{1 n} \\ -a_{22} & -a_{23} & \cdots & -a_{2 n} \\ \vdots & \vdots & & \vdots \\ -a_{n-1,2} & -a_{n-1,3} & \cdots & -\hat{a}_{n-1, n}\end{array}\right]+\epsilon \operatorname{det}\left[\begin{array}{cccc}-a_{12} & -a_{13} & \cdots & 0 \\ -a_{22} & -a_{23} & \cdots & 0 \\ \vdots & \vdots & & \vdots \\ -a_{n-1,2} & -a_{n-1,3} & \cdots & 1\end{array}\right]$

where the first determinant is zero by Lemma 2.1 and the second one is negative because $A$ is totally negative. Therefore, by Theorem $1.1, F_{\epsilon}$ is totally negative. Then by continuity

$$
\operatorname{det}\left[\begin{array}{cccc}
-a_{11} & -a_{13} & \cdots & -a_{1 n} \\
-a_{21} & -a_{23} & \cdots & -a_{2 n} \\
\vdots & \vdots & & \vdots \\
-a_{n-1,1} & -a_{n-1,3} & \cdots & -\hat{a}_{n-1, n}
\end{array}\right] \leq 0
$$

Denoting by $C_{1}$ and $C_{2}$ the cofactors of the element $-a_{n-1, n}$ in the left and right hand sides of equation (2), the left hand side of this equation can be written as

$$
\begin{aligned}
\operatorname{det}\left[\begin{array}{ccccc}
-a_{11} & -a_{13} & \ldots & -a_{1 n} & \\
-a_{21} & -a_{23} & \ldots & -a_{2 n} & \\
\vdots & \vdots & & \vdots \\
-a_{n-1,1} & -a_{n-1,3} & \ldots & \left(-a_{n-1, n}+\hat{a}_{n-1, n}\right)-\hat{a}_{n-1, n}
\end{array}\right] \\
=\left(-a_{n-1, n}+\hat{a}_{n-1, n}\right) C_{1}+\operatorname{det}\left[\begin{array}{cccc}
-a_{11} & -a_{13} & \ldots & -a_{1 n} \\
-a_{21} & -a_{23} & \ldots & -a_{2 n} \\
\vdots & \vdots & & \vdots \\
-a_{n-1,1} & -a_{n-1,3} & \ldots & -\hat{a}_{n-1, n}
\end{array}\right]
\end{aligned}
$$


and the right hand side can be written as

$$
\begin{array}{r}
\operatorname{det}\left[\begin{array}{cclcc}
-a_{12} & -a_{13} & \ldots & -a_{1 n} \\
-a_{22} & -a_{23} & \ldots & -a_{2 n} \\
\vdots & \vdots & & \vdots \\
-a_{n-1,2} & -a_{n-1,3} & \ldots & \left(-a_{n-1, n}+\hat{a}_{n-1, n}\right)-\hat{a}_{n-1, n}
\end{array}\right] \\
=\left(-a_{n-1, n}+\hat{a}_{n-1, n}\right) C_{2}+\operatorname{det}\left[\begin{array}{cccc}
-a_{12} & -a_{13} & \ldots & -a_{1 n} \\
-a_{22} & -a_{23} & \ldots & -a_{2 n} \\
\vdots & \vdots & & \vdots \\
-a_{n-1,2} & -a_{n-1,3} & \ldots & -\hat{a}_{n-1, n}
\end{array}\right] .
\end{array}
$$

Recall that $a_{n-1, n}<\hat{a}_{n-1, n}$ by Lemma 2.1, $C_{1}<C_{2}$ by induction hypothesis, the determinant in (4) is less than or equal to zero by (3) and the determinant in (5) is zero by Lemma 2.1. Then, the inequality (2) is true.

Note that the diagonal elements of $C(A)=A \circ A^{-T}$ and those of the matrix $A \circ A^{-1}$ are equal. Then, results referred to diagonal elements can be established for both matrices.

Theorem 2.1 Let $A=\left[-a_{i j}\right]$ be an $n \times n$ totally negative matrix. If $n \geq 3$, the diagonal entries $u_{i}$ of $C(A)$ satisfy: $u_{i}<0, i=1,2, \ldots, n$ and

$$
\begin{aligned}
u_{1} & >u_{2}, \\
u_{n-1} & <u_{n} .
\end{aligned}
$$

Proof: The negativity of the diagonal entries is evident, since $A$ is totally negative and

$$
u_{i}=-a_{i i} \frac{A_{i i}}{\operatorname{det} A}<0, \quad i=1,2, \ldots, n .
$$

Since $C(A)$ does not change if we multiply a row or a column by a positive number, we can assume that $a_{11}=a_{12}=a_{22}=1$ and then our problem is to show inequalities (6) and (7) for the matrix

$$
A=\left[\begin{array}{lll}
-1 & -1 & -A_{13} \\
-a_{21} & -1 & -A_{23} \\
-A_{31} & -A_{32} & -A_{33}
\end{array}\right]
$$


where $A_{i j}$ are the appropriate submatrices of $A$. Since $u_{1}=\frac{-1}{\operatorname{det}(A)} \operatorname{det}\left[\begin{array}{ll}-1 & -A_{23} \\ -A_{32} & -A_{33}\end{array}\right] \quad$ and $\quad u_{2}=\frac{-1}{\operatorname{det}(A)} \operatorname{det}\left[\begin{array}{ll}-1 & -A_{13} \\ -A_{31} & -A_{33}\end{array}\right]$ the proof of (6) reduces to prove the inequality

$$
\operatorname{det}\left[\begin{array}{ll}
-1 & -A_{13} \\
-A_{31} & -A_{33}
\end{array}\right]<\operatorname{det}\left[\begin{array}{ll}
-1 & -A_{23} \\
-A_{32} & -A_{33}
\end{array}\right] .
$$

If we apply Lemma 2.2 to the following totally negative submatrices of $A$

$$
\begin{gathered}
A^{\prime}=A[1,3 \mid 1,2,3]=\left[\begin{array}{lcl}
-1 & -1 & -A_{13} \\
-A_{31} & -A_{32} & -A_{33}
\end{array}\right] \\
A^{\prime \prime}=A[1,2,3 \mid 2,3]=\left[\begin{array}{ll}
-1 & -A_{13} \\
-1 & -A_{23} \\
-A_{32} & -A_{33}
\end{array}\right]
\end{gathered}
$$

we have

$$
\operatorname{det}\left[\begin{array}{ll}
-1 & -A_{13} \\
-A_{31} & -A_{33}
\end{array}\right]<\operatorname{det}\left[\begin{array}{ll}
-1 & -A_{13} \\
-A_{32} & -A_{33}
\end{array}\right]
$$

and

$$
\operatorname{det}\left[\begin{array}{ll}
-1 & -A_{13} \\
-A_{32} & -A_{33}
\end{array}\right]<\operatorname{det}\left[\begin{array}{ll}
-1 & -A_{23} \\
-A_{32} & -A_{33}
\end{array}\right]
$$

Then, the inequality (8) holds.

To prove inequality (7), we use the following: if $A=\left[-a_{i j}\right]$ is an $n \times n$ totally negative matrix and $J$ is the $n \times n$ anti-identity matrix, the matrix $J A J$ is also totally negative. Then, since

$$
\operatorname{diag}(J A J)=\left(-a_{n n},-a_{n-1, n-1}, \ldots,-a_{11}\right),
$$

applying (6) to $J A J$ we have $u_{n}>u_{n-1}$ and the proof is completed.

Example 1 Let the t.n. symmetric matrix

$$
A=\left[\begin{array}{ccc}
-20 & -16 & -4 \\
-16 & -12.2 & -2.8 \\
-4 & -2.8 & -0.2
\end{array}\right]
$$


Then

$$
A \circ A^{-1}=C(A)=\left[\begin{array}{rrr}
-27 & 32 & -4 \\
32 & -36.6 & 5.6 \\
-4 & 5.6 & -0.6
\end{array}\right]
$$

where $u_{1}=-27, u_{2}=-36.6,-u_{3}=-0.6$ and the inequalities of Theorem 2.1 are satisfied.

Example 2 Given the t.n. nonsymmetric matrix

$$
B=\left[\begin{array}{cccc}
-1 & -2 & -3 & -4 \\
-15 & -15 & -15 & -15 \\
-15 & -14 & -12 & -8 \\
-30 & -27 & -20 & -3
\end{array}\right]
$$

then

$$
\operatorname{diag}\left(B \circ B^{-1}\right)=\operatorname{diag}(C(B))=(-3,-110.5,-108,-1.5)
$$

and so, the inequalities of Theorem 2.1 hold.

Note that this example gives also examples of the same result for different $3 \times 3$ t.n. matrices.

\section{Sequence of diagonal entries}

Now let us formulate our problem: find necessary and sufficient conditions for an ordered $n$-tuple of real numbers to be the ordered n-tuple of the diagonal entries of $C(A)$ if $A$ is a totally negative $n \times n$ matrix. Let us proceed in the same way as in [11], so we shall study and prove the result in the $3 \times 3$ symmetric and nonsymmetric cases.

\subsection{Symmetric case}

Lemma 3.1 A $3 \times 3$ symmetric matrix is totally negative if and only if it is positively diagonally congruent to the matrix

$$
T=\left[\begin{array}{ccc}
-1 & -x_{3} & -x_{2} \\
-x_{3} & -1 & -x_{1} \\
-x_{2} & -x_{1} & -1
\end{array}\right]
$$


where $x_{1}, x_{2}, x_{3}$ are positive numbers satisfying

$$
\begin{aligned}
x_{i}>1, \quad i=1,2,3, & \\
& x_{2}>x_{1} x_{3} \quad \text { and } \\
-1-2 x_{1} x_{2} x_{3}+x_{1}^{2}+x_{2}^{2}+x_{3}^{2}=\operatorname{det} T<0 . &
\end{aligned}
$$

Proof: $\quad(\Rightarrow)$ Let $A=\left[-a_{i j}\right]$ be a $3 \times 3$ symmetric totally negative matrix and let $D$ be the positive diagonal matrix

$$
D=\left[\begin{array}{lcr}
\sqrt{a_{11}} & 0 & 0 \\
0 & \sqrt{a_{22}} & 0 \\
0 & 0 & \sqrt{a_{33}}
\end{array}\right]
$$

Then, $A=D T D$, where

$$
T=\left[\begin{array}{lcr}
-1 & -\frac{a_{12}}{\sqrt{a_{11} a_{22}}} & -\frac{a_{13}}{\sqrt{a_{11} a_{33}}} \\
-\frac{a_{12}}{\sqrt{a_{11} a_{22}}} & -1 & -\frac{a_{23}}{\sqrt{a_{22} a_{33}}} \\
-\frac{a_{13}}{\sqrt{a_{11} a_{33}}} & -\frac{a_{23}}{\sqrt{a_{22} a_{33}}} & -1
\end{array}\right] .
$$

Therefore, $A$ is positively diagonally congruent to a matrix $T$ as in (9), where

$$
x_{1}=\frac{a_{23}}{\sqrt{a_{22} a_{33}}}, \quad x_{2}=\frac{a_{13}}{\sqrt{a_{11} a_{33}}}, \quad x_{3}=\frac{a_{12}}{\sqrt{a_{11} a_{22}}}
$$

are positive numbers.

Since $A$ is totally negative $A_{11}=a_{22} a_{33}-\left(a_{23}\right)^{2}<0$, then $a_{23}>\sqrt{a_{22} a_{33}}$ and $x_{1}>1$. Similarly, we conclude that $x_{2}>1$ and $x_{3}>1$ since $A_{22}<0$ and $A_{33}<0$ respectively. Then condition (10) holds.

On the other hand, $A_{31}=a_{12} a_{23}-a_{22} a_{13}<0$ implies that $a_{13}>\frac{a_{12} a_{23}}{a_{22}}$ and $\frac{a_{13}}{\sqrt{a_{11} a_{33}}}>\frac{a_{12} a_{23}}{a_{22} \sqrt{a_{11} a_{33}}}$. As a consequence

$$
\frac{a_{13}}{\sqrt{a_{11} a_{33}}}>\frac{a_{23}}{\sqrt{a_{22} a_{33}}} \cdot \frac{a_{12}}{\sqrt{a_{11} a_{22}}}
$$

then $x_{2}>x_{1} x_{3}$ and condition (11) is true.

Finally, inequality (12) holds because $\operatorname{det} T=\frac{\operatorname{det} A}{(\operatorname{det} D)^{2}}$ and $A$ is totally negative. 
$(\Leftarrow)$ Since we have to prove that $D T D$ is t.n. for a positive diagonal matrix $D$, it is enough to prove that the matrix $T$ given in (9) satisfying conditions (10) - (12) is a t.n. matrix. All $1 \times 1$ and $3 \times 3$ minors are negative by (10) and (12) inequalities. Denote by $T_{i j}$ the corresponding $2 \times 2$ minors of $T$. The $2 \times 2$ minors corresponding to diagonal entries satisfy $T_{i i}=1-x_{i}{ }^{2}<0$ by (10). Now we need only to show that $2 \times 2$ minors $T_{i j}$ for $i \neq j$ are negative. Since $T$ is symmetric, $T_{13}=T_{31}=x_{1} x_{3}-x_{2}<0$ by (11). The minors $T_{12}=T_{21}=x_{3}-x_{1} x_{2}$ are negative since

$$
x_{2}>x_{1} x_{3} \text { and } x_{1}>1 \quad \Rightarrow \quad x_{1} x_{2}>x_{1}^{2} x_{3}>x_{3} .
$$

Finally, $T_{23}=T_{32}=x_{1}-x_{2} x_{3}<0$ can be obtained by multiplying (11) by $x_{3}>1$.

Note that inequality (11) in Lemma 3.1 can be replaced by $x_{1} x_{2}>x_{3}$ or by $x_{2} x_{3}>x_{1}$.

Theorem 3.1 The necessary and sufficient condition for three negative numbers $u_{1}, u_{2}$ and $u_{3}$, be the diagonal entries of the combined matrix of a $3 \times 3$ symmetric totally negative matrix $A$ is

$$
u_{1}+u_{3}-u_{2}-1>0
$$

Proof: Let $A$ be a $3 \times 3$ symmetric totally negative matrix. By Lemma $3.1 A$ is positively diagonally congruent to the matrix $T$ of (9). Since $C(D A)=C(A D)=C(A)$ for any nonsingular diagonal matrix $D$, it follows that $C(A)=C(T)$. Thus we can rewrite our theorem in terms of $T$. Note that the diagonal entries of $C(T)$ are

$$
u_{1}=\frac{-\left(1-x_{1}^{2}\right)}{\operatorname{det} T}, \quad u_{2}=\frac{-\left(1-x_{2}^{2}\right)}{\operatorname{det} T}, \quad u_{3}=\frac{-\left(1-x_{3}^{2}\right)}{\operatorname{det} T},
$$

and then

$$
u_{1}+u_{3}-1-u_{2}=\frac{1}{\operatorname{det} T}\left(2 x_{2}\right)\left(x_{1} x_{3}-x_{2}\right) \text {. }
$$

$(\Rightarrow)$ By equation (15) we have $u_{1}+u_{3}-1-u_{2}>0$ since $\operatorname{det} T<0$, $x_{1} x_{3}<x_{2}$ and $x_{2}>0$. 
$(\Leftarrow)$ Note that $x_{i}{ }^{2}=1+u_{i} \operatorname{det} T, i=1,2,3$ by equations (14). Adding these values on equation (12), we have

$$
\begin{aligned}
\operatorname{det} T= & -1-2 \sqrt{\left(1+u_{1} \operatorname{det} T\right)\left(1+u_{2} \operatorname{det} T\right)\left(1+u_{3} \operatorname{det} T\right)} \\
& +\left(1+u_{1} \operatorname{det} T\right)+\left(1+u_{2} \operatorname{det} T\right)+\left(1+u_{3} \operatorname{det} T\right),
\end{aligned}
$$

which can be written as

$$
\begin{array}{r}
4(\operatorname{det} T)^{2} u_{1} u_{2} u_{3}+\operatorname{det} T\left[4\left(u_{1} u_{2}+u_{1} u_{3}+u_{2} u_{3}\right)\right. \\
\left.-\left(u_{1}+u_{2}+u_{3}-1\right)^{2}\right]+4=0
\end{array}
$$

Let us see that this second degree equation written in brief as $a(\operatorname{det} T)^{2}+$ $b(\operatorname{det} T)+c=0$ has a negative root. Its discriminant satisfies $b^{2}-4 a c>b^{2}$, since $-4 a c=-64 u_{1} u_{2} u_{3}>0$. Therefore always $-b+\sqrt{b^{2}-4 a c}>0$. Then

$$
\frac{-b+\sqrt{b^{2}-4 a c}}{2 a}<0
$$

since $a=4 u_{1} u_{2} u_{3}<0$. Hence there exists a solution satisfying $\operatorname{det} T<0$.

Coefficients $x_{i}$ satisfy $x_{i}>1$ since $\operatorname{det} T<0$ and $u_{i}<0$. Then inequalities in (10) hold.

Moreover, by (15), we obtain $x_{1} x_{3}-x_{2}<0$ since $\operatorname{det} T<0$ and $x_{2}>0$. Then (11) is true.

Example 3 It is easy to see that for the following $3 \times 3$ t.n. symmetric matrix $A$, the diagonal entries of $C(A)$ satisfy equation (13):

$$
A=\left[\begin{array}{lll}
-1 & -3 & -8 \\
-3 & -1 & -2 \\
-8 & -2 & -1
\end{array}\right] \rightarrow C(A)=\left[\begin{array}{ccc}
-0.15 & 1.95 & -0.8 \\
1.95 & -3.25 & 2.2 \\
-0.8 & 2.2 & -0.4
\end{array}\right]
$$

where $\left(u_{1}+u_{3}-1\right)=-1.55>-3.15=u_{2}$.

Now let us see a converse example.

Example 4 Given $u_{1}=-2, u_{2}=-8, u_{3}=-4$ such that $u_{1}+u_{3}-u_{2}-1=$ $1>0$, we will find a symmetric t.n. matrix $T$ such that $\operatorname{diag}(C(T))=$ $\left(u_{1}, u_{2}, u_{3}\right)$. 
Replacing the given values in (16) it is obtained

$$
-256(\operatorname{det} T)^{2}-\operatorname{det} T+4=0 .
$$

This equation has a solution $\operatorname{det} T<0$, required by the theorem,

$$
\operatorname{det} T=-\frac{1+\sqrt{4097}}{512} \approx-0.1269684
$$

Using equations $x_{i}=\sqrt{1+u_{i} \operatorname{det} T}, i=1,2,3$ we have

$$
x_{1} \approx 1.1197932, \quad x_{2} \approx 1.4197701, \quad x_{3} \approx 1.227955,
$$

which satisfy $x_{1} x_{3}-x_{2}<0$. Then, the conditions of Lemma 3.1 are satisfied and $T$ is a t.n. matrix. The combined matrix of $T$ is

$$
C(T)=\left[\begin{array}{rrr}
-2 & 3.5 & -0.5 \\
3.5 & -8 & 5.5 \\
-0.5 & 5.5 & -4
\end{array}\right]
$$

where $\operatorname{diag}(C(T))=(-2,-8,-4)$, just as we expected.

\subsection{Nonsymmetric case}

Let us turn now to the case of a general totally negative $3 \times 3$ matrix. First we prove the next lemma.

Lemma 3.2 A $3 \times 3$ matrix $A$ is totally negative if and only if it is positively diagonally equivalent to the matrix

$$
T=\left[\begin{array}{ccc}
-\frac{v_{1}}{v_{1}+1} & -1 & -p \\
-1 & -1 & -1 \\
-q & -1 & -\frac{v_{3}}{v_{3}+1}
\end{array}\right]
$$

where

$$
\begin{gathered}
v_{i}>0, \quad i=1,3, \\
p, q>1, \\
\operatorname{det} T=\frac{(p-1)(q-1)\left(v_{1}+1\right)\left(v_{3}+1\right)-1}{\left(v_{1}+1\right)\left(v_{3}+1\right)}<0 .
\end{gathered}
$$


Proof: $\quad(\Rightarrow)$ Let $A=\left[-a_{i j}\right]$ a $3 \times 3$ totally negative matrix. Constructing

$$
T=\left[\begin{array}{llr}
\frac{-a_{11} a_{22}}{a_{12} a_{21}} & -1 & \frac{-a_{13} a_{22}}{a_{12} a_{23}} \\
-1 & -1 & -1 \\
\frac{-a_{31} a_{22}}{a_{32} a_{21}} & -1 & \frac{-a_{33} a_{22}}{a_{32} a_{23}}
\end{array}\right]
$$

and

$$
D_{1}=\left[\begin{array}{ccr}
a_{12} & 0 & 0 \\
0 & a_{22} & 0 \\
0 & 0 & a_{32}
\end{array}\right], \quad D_{2}=\left[\begin{array}{ccc}
\frac{a_{21}}{a_{22}} & 0 & 0 \\
0 & 1 & 0 \\
0 & 0 & \frac{a_{23}}{a_{22}}
\end{array}\right]
$$

we have that $A=D_{1} T D_{2}$. Taking

$$
v_{1}=\frac{a_{11} a_{22}}{a_{12} a_{21}-a_{11} a_{22}}, v_{3}=\frac{a_{33} a_{22}}{a_{32} a_{23}-a_{33} a_{22}}, p=\frac{a_{13} a_{22}}{a_{12} a_{23}} \text { and } q=\frac{a_{31} a_{22}}{a_{21} a_{23}},
$$

the matrix $T$ has the form (17).

As $A$ is a totally negative matrix, $A_{33}<0$. This implies $a_{11} a_{22}-a_{21} a_{12}<0$ thus $v_{1}>0$. In a similar way, $A_{11}<0$ implies $a_{33} a_{22}-a_{32} a_{23}<0$, then $v_{3}>0$. Moreover, since $A_{31}=a_{12} a_{23}-a_{13} a_{22}<0$ we have $p>1$. From $A_{13}<0$ we obtain $q>1$. Then, conditions (18) and (19) hold.

Finally, it is easy to check that condition (20) corresponds to $\operatorname{det} T$ and $\operatorname{det} T<0$ since $\operatorname{det} A=\operatorname{det} D_{1} \operatorname{det} T \operatorname{det} D_{2}<0$ and $A$ is t.n.

$(\Leftarrow)$ We need to show that if $A$ is positively diagonally equivalent to $T$ satisfying the corresponding conditions, then $A$ is totally negative. Our proof reduces to show that $T$ is totally negative.

By conditions (18) to (20), entries $t_{i j}$ and $\operatorname{det} T$ are negative. If we define

$$
\frac{v_{1}}{v_{1}+1}=r \quad \text { and } \quad \frac{v_{3}}{v_{3}+1}=s,
$$

then matrix $T$ has the structure

$$
T=\left[\begin{array}{lll}
-r & -1 & -p \\
-1 & -1 & -1 \\
-q & -1 & -s
\end{array}\right]
$$

where $0<r, s<1$ and $p, q>1$. From here is clear that all minors of $T$ of order 2 are negative and then $T$ is t.n. 
Theorem 3.2 The necessary and sufficient condition for three negative numbers $u_{1}, u_{2}$ and $u_{3}$, be the diagonal entries of the combined matrix of a $3 \times 3$ totally negative matrix $A$ is

$$
u_{1}+u_{3}-u_{2}-1>0
$$

Proof: Let $A$ be a $3 \times 3$ totally negative matrix. By Lemma $3.1 A$ is positively diagonally equivalent to a matrix $T$ as in (17). Considering that $C(D A)=C(A D)=C(A)$ for all nonsingular diagonal matrix $D$, it follows that $C(A)=C(T)$. Then, we assume that $A=T$.

$(\Rightarrow)$ With the above consideration we will prove that given $T$ defined by (17) and satisfying conditions (18), (19), (20) and $\operatorname{diag}(C(T))=\left(u_{1}, u_{2}, u_{3}\right)$, then $u_{1}+u_{3}-u_{2}-1>0$.

First, we denote the diagonal entries of $T^{-1}$ by $\left(\hat{a}_{11}, \hat{a}_{22}, \hat{a}_{33}\right)$ and by $d$ the numerator of the fraction (20), Then

$$
\begin{gathered}
\hat{a}_{11}=\frac{1}{\operatorname{det} T}\left(\frac{v_{3}}{v_{3}+1}-1\right)=\frac{1}{\operatorname{det} T}\left(\frac{-1}{v_{3}+1}\right)=\frac{-\left(v_{1}+1\right)}{d}, \\
\hat{a}_{22}=\frac{1}{\operatorname{det} T}\left(\frac{v_{1} v_{3}}{\left(v_{1}+1\right)\left(v_{3}+1\right)}-p q\right)=\frac{v_{1} v_{3}-p q\left(v_{1}+1\right)\left(v_{3}+1\right)}{d}, \\
\hat{a}_{33}=\frac{1}{\operatorname{det} T}\left(\frac{v_{1}}{v_{1}+1}-1\right)=\frac{-\left(v_{3}+1\right)}{d} .
\end{gathered}
$$

Thus, the diagonal entries of $C(A)=A \circ\left(A^{-T}\right)$ are

$$
u_{1}=\frac{v_{1}}{d}, u_{2}=-\frac{v_{1} v_{3}-p q\left(v_{1}+1\right)\left(v_{3}+1\right)}{d}, u_{3}=\frac{v_{3}}{d} .
$$

Replacing equations (22) in the left part of condition (21), we have

$$
u_{1}+u_{3}-u_{2}-1=\frac{1}{d}\left(v_{1}+v_{3}+v_{1} v_{3}-p q\left(v_{1}+1\right)\left(v_{3}+1\right)-d\right) .
$$

Taking into account $v_{1}+v_{3}+v_{1} v_{3}=\left(v_{1}+1\right)\left(v_{3}+1\right)-1$, we have

$$
u_{1}+u_{3}-u_{2}-1=\frac{1}{d}\left(v_{1}+1\right)\left(v_{3}+1\right)(1-p q-(p-1)(q-1))
$$

is a positive number by conditions (18)-(20). 
$(\Leftarrow)$ Now we will prove that if $u_{1}, u_{2}, u_{3}$ are negative numbers such that

$$
u_{1}+u_{3}-u_{2}-1>0
$$

then we can built a matrix $T$ with the structure (17) satisfying (18)-(20) such that $u_{1}, u_{2}, u_{3}$ are the diagonal entries of $C(T)$. That is, we are going to prove that the system

$$
u_{1}=\frac{v_{1}}{d}, \quad u_{2}=-\frac{v_{1} v_{3}-p q\left(v_{1}+1\right)\left(v_{3}+1\right)}{d}, \quad u_{3}=\frac{v_{3}}{d},
$$

has the expected solutions.

Observe that $u_{2} d=p q\left(v_{1}+1\right)\left(v_{3}+1\right)-v_{1} v_{3}$ implies

$$
p q=\frac{u_{2} d+u_{1} u_{3} d^{2}}{\left(u_{1} d+1\right)\left(u_{3} d+1\right)},
$$

and this implies

$$
\frac{1}{p} \frac{1}{q}=\frac{\left(u_{1} d+1\right)\left(u_{3} d+1\right)}{u_{2} d+u_{1} u_{3} d^{2}} .
$$

From definition of $d$, it follows

$$
(p-1)(q-1)=\frac{(d+1)}{\left(u_{1} d+1\right)\left(u_{3} d+1\right)} .
$$

From equation (24) it follows

$$
\left(u_{1} d+1\right)\left(u_{3} d+1\right)=\frac{u_{2} d+u_{1} u_{3} d^{2}}{p q} .
$$

Replacing (26) in (25), we have

$$
(p-1)(q-1)=\frac{(d+1) p q}{u_{2} d+u_{1} u_{3} d^{2}} .
$$

Finally

$$
\left(1-\frac{1}{p}\right)\left(1-\frac{1}{q}\right)=\frac{d+1}{u_{2} d+u_{1} u_{3} d^{2}} .
$$

Our system of equations (23) has been reduced to equations (24) and (27).

It is well known that a necessary and sufficient condition for the system of equations $x y=u,(1-x)(1-y)=v$ has solutions $x, y \in] 0,1[$ is that 
$\sqrt{u}+\sqrt{v} \leq 1$. Using this equivalence with $x=\frac{1}{p}, y=\frac{1}{q}$, the system given by equations (24) and (27) has solutions in ]0,1[ if and only if

$$
\sqrt{\frac{\left(u_{1} d+1\right)\left(u_{3} d+1\right)}{u_{2} d+u_{1} u_{3} d^{2}}}+\sqrt{\frac{d+1}{u_{2} d+u_{1} u_{3} d^{2}}} \leq 1 .
$$

If we replace $d=-1$ in left side of (28) and use equation (23) we have

$$
\sqrt{\frac{\left(-u_{1}+1\right)\left(-u_{3}+1\right)}{-u_{2}+u_{1} u_{3}}}=\sqrt{\frac{\left(v_{1}+1\right)\left(v_{3}+1\right)}{p q\left(v_{1}+1\right)\left(v_{3}+1\right)-v_{1} v_{3}+v_{1} v_{3}}}=\sqrt{\frac{1}{p q}}<1 .
$$

This inequality is strict since $u_{1}+u_{3}-u_{2}-1>0$ implies $\left(1-u_{1}\right)\left(1-u_{3}\right)<$ $-u_{2}+u_{1} u_{3}$. Thus, there exists an small enough $0<\varepsilon<1$ such that for $d=-1+\varepsilon$ condition (28) holds. Then, there exist $p, q>1$ satisfying the system given by (24) and (27). This implies $v_{1}=d u_{1}>0, v_{3}=d u_{3}>0$ and $\operatorname{det} T=\frac{d}{\left(v_{1}+1\right)\left(v_{3}+1\right)}<0$.

The following example illustrates this last theorem.

Example 5 Let $A$ be the $3 \times 3$ nonsingular nonsymmetric totally negative matrix and $A^{-1}$ its inverse

$$
A=\left[\begin{array}{ccc}
-8 & -14 & -19 \\
-13 & -20 & -27 \\
-17 & -26 & -35
\end{array}\right], \quad A^{-1}=\left[\begin{array}{ccc}
1 & -2 & 1 \\
-2 & 21.5 & -15.5 \\
1 & -15 & 11
\end{array}\right]
$$

The combined matrix of $A$ is

$$
C(A)=\left[\begin{array}{ccc}
-8 & 28 & -19 \\
26 & -430 & 405 \\
-17 & 403 & -385
\end{array}\right]
$$

As we can see the diagonal entries are negative numbers and satisfy the required condition.

Now let us see a converse example. 
Example 6 Given negative numbers $u_{1}=-2, u_{2}=-8, u_{3}=-3$ satisfying condition $u_{1}+u_{3}-u_{2}-1>0$, we will find a matrix $T$ such that $\operatorname{diag}(C(T))=$ $\left(u_{1}, u_{2}, u_{3}\right)$.

Replacing the given values in equation (28) the value $d=-\frac{3-\sqrt{201}}{12} \approx$ -0.931454 gives the equality. So, taking a smaller value for example, $d=$ -0.94 we obtain $v_{1}$ and $v_{3}$. Using the equation (24) with the condition $p, q>1$ we can obtain $p$ and $q$, for instance, $p=1.00527$ and $q=1.1604$.

With these values we built the matrix

$$
T=\left[\begin{array}{rrr}
-0.66443 & -1 & -1.00527 \\
-1 & -1 & -1 \\
-1.1604 & -1 & -0.748111
\end{array}\right]
$$

and its combined matrix

$$
C(T)=\left[\begin{array}{rrr}
-2 & 4.92693 & -1.92693 \\
3.07307 & -8 & 5.92693 \\
-0.0730739 & 4.07307 & -3
\end{array}\right]
$$

has the given diagonal entries.

\section{References}

[1] T. Ando. Totally Positive Matrices. Linear Algebra Appl., 90(1987), pp. $165-219$.

[2] E. Bristol. Interaction analysis. IEEE Trans. on Automatic Control, AC11(1966), pp. 133-134.

[3] R. Bru, M. Gassó, I. Giménez and M. Santana. Nonnegative combined matrices. J. Appl. Math., 2014(2014), Article ID 182354. (doi: 10.1155/2014/182354).

[4] R. Bru, M. Gassó, I. Giménez and M. Santana. Combined matrices of sign regular matrices. Linear Algebra Appl., 498(2016), pp. 88-98. (doi: 10.1016/j.laa.2014.12.010).

[5] R. Bru, M. Gassó, I. Giménez and J. A. Scott. The Hadamard product of a nonsingular general H-Matrix and its inverse transpose is diagonally dominant. J. of Appl. Math., 2015(2015), Article ID 264680. (doi: 10.1155/2015/264680). 
[6] R. Canto, B. Ricarte and A. Urbano. Some characterizations of totally nonpositive (totally negative) matrices. Electron. J. Linear Algebra, 20(2010), pp. 241-253.

[7] S. Fallat and P. van den Driesche. On matrices with all minors negative. Electron. J. Linear Algebra, 7(2000), pp. 92-99.

[8] M. Fiedler. Relations between the diagonal entries of an M-matrix and of its inverse. Mat.-fyz.casopis SAV, 12(1962), pp. 123-128.

[9] M. Fiedler. Relations between the diagonal entries of two mutually inverse positive definite matrices. Czechoslovak Math. J., 14(1964), pp. $39-51$.

[10] M. Fiedler., T.L. Markham. Consecutive- column and row properties of matrices and the Loewner -Neville factorization. Linear Algebra Appl., 266(1997), pp. 243-259.

[11] M. Fiedler and T. L. Markham. Combined matrices in special classes of matrices. Linear Algebra Appl., 435(2011), pp. 1945-1955.

[12] M.Fiedler, F.J.Hall. G-matrices. Linear Algebra Appl., 436(2012), pp. 731-741.

[13] R. A. Horn and C. R. Johnson, Topics in Matrix Analysis, Cambridge Univ. Press, 1991.

[14] J.J. McDonald, M. Neuman, H. Schneider and M.J. Tsatsomeros. Inverse $\mathrm{M}$ matrix inequalities and generalized ultrametric matrices. Linear Algebra Appl., 220(1995), pp. 329-349.

[15] J. M. Peña. Tests for the recognition of total positivity. SeMA Journal, 62(2013), pp. 61-73. (doi.org/ 10.1007/s40324-013-0008-z)

[16] R. S. Varga, Matrix Iterative Analysis, (Prentice Hall, Inc., Englewood Cliffs, New Jersey, 1962.) Second Ed. Springer, Springer Series in Computational Mathematics, 27, Berlin, 2000. 